\title{
Fusobacterium Nucleatum Septicemia Associated with Hematologic Diseases
}

\author{
Hisashi Funada, M D and Ken-ichi Hattori, MD \\ The Third Department of Internal Medicine, \\ Kanazawa University School of Medicine \\ Naomi Nishida, M D \\ Department of Pediatrics, Kanazawa University School of Medicine
}

\begin{abstract}
For the past 2 years Fusobacterium nucleatum septicemia has been observed in our clinic in 2 patients with hematologic diseases: chronic myelogenous leukemia in blastic transformation and acute lymphocytic leukemia in the second relapse. These patients were treated with cytotoxic agents and corticosteroids, and had both severe pancytopenia and foul-smelling oral ulcerations preceding the onset. Evidence of thrombophlebitis, embolic phenomena or metastatic abscesses was lacking in both cases. The clinical picture of fusobacterial septicemia reported here did not seem to vary from that of sepsis caused by any gramnegative rod. In addition, these patients developed another gram-negative septicemia on 2 successive occasions after the onset of fusobacterial septicemia, and died. Extensive survey of the literature revealed that very few cases of fusobacterial septicemia have been published in this country. Wider use of advanced anaerobic blood culture method and identification techniques is expected to provide a higher incidence of septicemia with this organism than so far reported.
\end{abstract}

Key Words: Chronic myelogenous leukemia and acute lymphocytic leukemia

Bacteremias due to gram-negative nonsporeforming anaerobic rods, collectively referred to as 'Bacteroides' in most series, have recently assumed greater clinical importance in patients with depressed resistance $^{1-6)}$. Consequently, speciation of these organisms has become important in order to define the exact pathogenic significace of each species and because of the difference in antimicrobial susceptibility found from one species to another ${ }^{4,7)}$.

Received for publication September 30, 1976, Present address and reprint request to: Hisashi

Funada, The Third Department of Internal

Medicine, Kanazawa University, School of

Medicine, 13-1, Takara Machi, Kanazawa, Ishikawa Prefecture, P. O. 920, Japan
Fusobacterial septicemia has been rather rarely reported in the past ${ }^{8-10)}$, partly because fusobacteria, especially Fusobacterium nucleatum, are more difficult to isolate and maintain than any others ${ }^{7)}$.

In this report we describe 2 cases of $\mathrm{F}$. nucleatum septicemia complicating hematologic diseases.

\section{CASE REPORTS}

Case 1: A 50-year-old farmer with chronic myelogenous leukemia (CML) of about 2 years' duration was admitted for the second time to our clinic on May 22, 1974 , with a low-grade fever of 37 to $38^{\circ} \mathrm{C}$, massive hepatosplenomegaly, white blood cell count of $23,100 / \mathrm{mm}^{3}$, composed of $64 \%$ 
myeloblasts and hemoglobin level of $5.8 \mathrm{~g} /$ dl. The bone marrow revealed morphology consistent with GML in blastic transformation. Therapy for blastic crisis consisted of cyclocytidine, daunorubicin, 6-mercaptopurine and prednisolone. Blood transfusions were used in accordance with the degree of anemia. He responded poorly to this systemic therapy, which resulted in leukopenia of below $1,500 / \mathrm{mm}^{3}$ one month later. A foul-smelling and undermining ulceration with firm and ragged edges appeared on the tongue. The ulcer was about $1.0 \mathrm{~cm}$ in diameter, covered with a dirty-gray coat. It was thought to be dut to leukemic infiltration. He complained of severe pain on swallowing, and developed increasing fevers. On his 34th hospital day he complained of the sudden onsent of palpitation and shortness of breath. Temperature rose to $39.3^{\circ} \mathrm{C}$. Blood pressure was normotensive. Soon after 5 separate blood cultures were obtained, antimicrobial therapy was instituted with cefazolin and kanamycin in combination. Four days later, bacteriology laboratory reported that 2 of the 5 blood cultures had grown anaerobic gram-negative rods, which were subsequently identified as F. nucleatum, sensitive by disc to cefazolin, not to kanamycin. Therapy was switched to cefazolin alone. On this regimen he clinically improved and his temperature, although continually elevated, became lower. But, coincident with an increase in the white blood cell counts to more than $100,000 / \mathrm{mm}^{3}$ with 80 to $90 \%$ myeloblasts, daily temperature elevations as high as 39 to $40^{\circ} \mathrm{C}$ recurred. Any antileukemic therapy proved to be unsuccessful. Two weeks after the onset of $F$. nucleatum septicemia, another gram-negative septicemia developed with a resistant Enterobacter cloacae, followed in 3 days by Pseudomonas aeruginosa. He died on July 11, 1974.

Case 2: A 7-year-old boy with acute lymphocytic leukemia (ALL) of 2 years' duration was admitted to our clinic on March 8, 1976, because of nasal bleeding. Three months previously he had been treated for systemic relapse of this disease. Physi- cal examination on admission revealed an ill-appearing pale boy with nasal bleeding. Blood pressure was $90 / 0 \mathrm{mmHg}$, pulse rate $140 / \mathrm{min}$, respiratory rate $24 / \mathrm{min}$ and temperature $37.3^{\circ} \mathrm{C}$. He had no lymphadenopathy, hepatosplenomegaly nor signs of an acute infectious process. Hemoglobin was $6.4 \mathrm{~g} / \mathrm{dl}$, hematocrit $18 \%$, platelet count below $2,000 / \mathrm{mm}^{3}$, and leukocyte count $20,800 /$ $\mathrm{mm}^{3}$ with $88 \%$ blast forms, $1 \%$ promyelocytes, $1 \%$ bands and 10\% lymphocytes. Bone marrow studies showed 80\% blast forms. Therapy for relapse was instituted with adriamycin, cytosine arabinoside, vincristine and prednisolone. Blood transfusions were also performed according to the degree of anemia. Soon thereafter, he developed increasing fevers and several ulcerating lesions with slight bleeding were noted on the tengue and gum. They were fairly deep and of small size: about $0.5 \mathrm{~cm}$ in diameter. Three leukocyte counts after anti-leukemic therapy showed below 500/ $\mathrm{mm}^{3}$. On his 11th hospital day, spiking fever up to $40.4^{\circ} \mathrm{C}$ developed, and nausea, vomiting and melena ensued. Two blood cultures obtained at the temperature spike grew anaerobic gram-negative rods, subsequently identified as F. nucleatum. He was promptly placed on cephalothin without defervescence. Five days later, therefore, antimicrobial therapy was switched to lincomycin. He, although febrile, did not seem to be in distress. On his 19th hospital day, he had chills and a temperature spike of $40.8^{\circ} \mathrm{C}$ again. Two blood cultures drawn at this time revealed Klebsiella, sensitive by disc to both gentamicin and cephaloridine, which led to some clinical improvement. There was some extension of the oral ulcerating lesions, from which Klebsiella was cultured in abundance. Melena occurred several times. He became quite anorectic. Severe pancytopenia persisted, and the bone marrow was almost completely replaced by blast cells. Therapy was instituted with L-asparaginase, vincristine and dexamethasone, which did not bring about any improvement in hematologic status. On his 37 th hospital day, shaking chills and spiking 
fevers developed again, and blood pressure fell down. At this time 2 separate blood cultures grew enterococci and Klebsiella that showed different characteristics and antimicrobial susceptibility pattern from the former Klebsiella, respectively. The next day, April 14, 1976, he died.

\section{BACTERIOLOGY}

Blood cultures were performed by inoculating venous blood directly into bottles containing brain heart infusion broth and thioglycollate medium (Culture Bottles No. 1 and No. 2 'Eiken', respectively) under partial vacuum. At least 2 blood cultures were performed before antimicrobial therapy was started. Cultures were incubated a $37^{\circ} \mathrm{C}$ for 2 weeks and inspected daily. Subculture and identification of aerobic isolates was routinely carried out by conventional $\operatorname{method}^{11)}$. In anaerobic cultivation, the anaerobic jar method was employed with an atomosphere of $80 \%$ nitrogen, $10 \%$ hydrogen and $10 \%$ carbon dioxide in the presence of 'Deoxo' room temperature catalyst. GAM agar and semisolid medium 'Nissui' were used for maintenance and as base media for biochemical tests unless otherwise noted. For identification of anaerobic isolates we referred to the criteria listed in the Anaerobe Laboratory Manual of the Virginia Polytechnic Institute Anaerobe Laboratory ${ }^{12}$. Susceptibility of anaerobic isolates to antimicrobial agents was determined by the disc method with commercial 3-graded concentration discs (Tridisks 'Eiken') as well as by the agar-plate dilution method, indicated as minimal inhibitory concentrations $(\mathrm{MIC})^{13)}$.

Blood cultures obtained from 2 cases reported here were positive for fusobacteria. Growth occurred in both Culture Bottles No. 1 and No. 2 after 48 to 72 hours' incubation. By Gram stain they were gramnegative, nonsporeforming, rod-shaped orgainsms with tapered ends and reddish staining granules. On anaerobic subculture to GAM agar plates, ivory-colored colonies appeared in 2 days, and revealed a flecked internal structure. The isolates from these 2 cases showed about the same biochemical reactions (Table), and were finally identified as F. nucleatum. Both isolates were highly sensitive by disc to ampicillin, car. benicillin, sulbenicillin, cephalothin, cephaloridine, cefazolin, chloramphenical, tetracycline, doxycycline, minocycline, colistin, polymyxin-B and lincomycin, but resistant

Table Biochemical reactions of 2 blood isolates from Cases 1 and 2 .

\begin{tabular}{|c|c|c|c|c|c|c|c|c|}
\hline Growth on: & & Milk & - & Acid produced & fror & m : & Ribose & - \\
\hline 'modified $\mathrm{FM}^{13}$ & + & Nitrate reduced ${ }^{*} 3$ & - & Arabinose & - & & Trehalose & - \\
\hline 'Bacteroides' 13) & - & Indole produced $* 3$ & + & Fructose & $w^{*} 6$ & & Salicin & - \\
\hline Gas & - & Gelatin 1 iquefied $*^{4}$ & - & Mannose & - & & Rhamnose & - \\
\hline Motility & - & Starch hydrolyzed & - & Glucose $\{W: C$ & 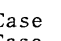 & 1 & Raffinose & - \\
\hline Hemolys is * 1 & - & Esculin hydrolyzed & - & Lactose & - & & Dulcitol & - \\
\hline End products ${ }^{13}$ ) & $A, P, B * 2$ & Lipase $*^{5}$ & - & Ma1tose & - & & Sorbitol & - \\
\hline Lactate $\rightarrow$ propionate ${ }^{14)}$ & - & Lecithinase $*^{5}$ & - & Mannitol & - & & Erythritol & - \\
\hline Threonine $\rightarrow$ propionate 13,14$)$ & + & & & Sucrose & - & & Inositol & - \\
\hline $20 \%$ bile-growth $^{13}$ ) & - & & & Xylose & - & & Starch & - \\
\hline
\end{tabular}

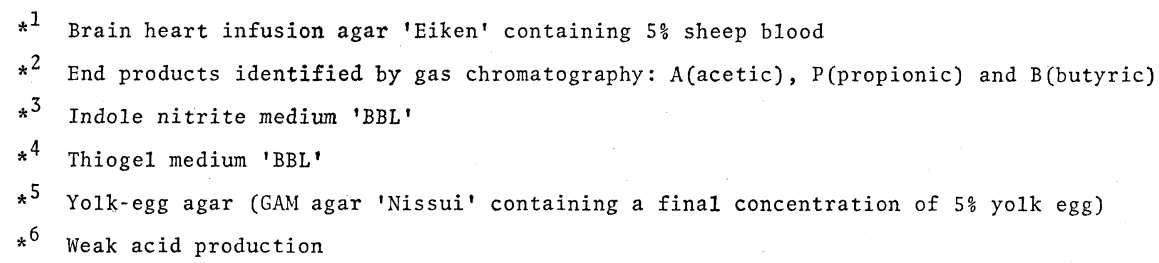


to streptomycin, kanamycin and gentamicin. MICs of antimicrobial agents to the isolate from Case 1 were as follows: ampicillin, cefazolin and lincomycin : $\leqq 0.20 \mu \mathrm{g} / \mathrm{ml}$, each, tetracycline and colistin: $0.39 \mu \mathrm{g} / \mathrm{ml}$, each, chloramphenicol: $0.78 \mu \mathrm{g} / \mathrm{ml}$, and streptomycin, kanamycin and gentamicin : $<100 \mathrm{~g} /$ $\mathrm{ml}$, each.

Unfortunately, dirty exudates seen at the base of oral ulcerative lesions were not examined by Gram stain, nor cultured anaerobically. The aerobic and facultative organisms isolated from the blood was also isolated simultaneously or beforehand from throat cultures in these cases.

\section{DISCUSSION}

Human infections due to gram-negative, nonsporeforming, anaerobic rods or 'Bacteroides' are uncommon, but have been reported with increasing frequency in recent years. In these infections, the Fusobacterium species, predominantly F. necrophorum and F. nucleatum, are found fairly frequently, although the Bacteroides species are the most commonly encountered. Many of the fusobacterial infections are associated with the oral cavity and respiratory tract ${ }^{4,15,16)}$, as might be expected from the fact that these organisms are part of constant inhabitants in the mouth ${ }^{17)}$. F. nucleatum is commonly found in infections of the oral cavity and the lungs, less frequently in genitourinary tract infections, and rarely in gastrointestinal tract infections ${ }^{4}$. In some cases they are primarily responsible either alone or in combination with other bacteria; in others their role is secondary ${ }^{18)}$. It is, therefore, sometimes impossible to determine their exact pathogenic significance.

About $10 \%$ of bacteremias in a general hospital have been reported to be caused by anaerobic bacteria, among which the 'Bacteroides' organisms are the most frequently recovered while the clostridia and anaerobic cocci are found less often ${ }^{5,19,20)}$. The Fusobacterium species are relatively uncommon pathogens encountered among 'Bacteroides' bacteremias. Felner and Dowell $^{4)}$ found 55 cases of fusobacterial bacteremia compared with 195 cases of bacteremia due to the Bacteroids species. In the study by Wilson et al. ${ }^{5}$, only 2 out of 52 patients with clinically significant 'Bacteroides' bacteremia had fusobacterial bacteremia (F. necrophorum and F. nucleatum, one case each). Also in the series of Ellner et al. ${ }^{20)}$, only 4 fusobcterial isolates (F. nucleatum 1 and other Fusobacterium species 3) were found among 63 'Bacteroides' isolates from the blood. In this country septicemias due to the Bacteroides species, particularly B. fragilis, have increased in frequency in recent years, while there have been very few published reports on cases of proved fusobacterial septicemia despite our extensive survey of the Japanese literature.

Among the 'Bacteroides' organisms, F. nucleatum was found more difficult to isolate and maintain than any other ${ }^{7)}$, probably because it is more exacting in their demand for growth factors than others ${ }^{18)}$, and because it is fairly readily killed by exposure to the air if not given some protection $^{21)}$. In this regard, improvement in anaerobic blood culture method and techniques of identification may provide a higher incidence of septicemia with this organism.

In addition, it is noteworthy that 'Bacteroides' bacteremias were likely to occur in patients with underlying disorders such as prior surgery, malignant neoplasms including leukemias or diabetes mellitus, or who had been treated with corticosteroids, cytotoxic agents, immunosuppressants or antimicrobial agents inactive against anaerobes $^{4)}$. Interestingly, Sinkovics and Smith ${ }^{3)}$, and Chow and Guze $^{6}$ noted that 'Bacteroides' bacteremias tend to complicate solid tumors rather than hematologic malignancies which are important predisposing conditions for aerobic gram-negative sepsis.

As Klainer and Beisel ${ }^{22)}$ indicated, therefore, it may be possible to conclude that fusobacteria are typical of the opportunistic organisms encountered when host defenses are defective. Moreover, it is well-known that the severity of infectious process is correlated with impaired host defenses ${ }^{6,233}$. 
Two cases reported here had GML in blastic transformation and ALL in relapse, respectively, and prior to the onset of fusobacterial septicemia they had received energetic antileukemic therapy with cytotoxic agents and corticosteroids, resulting in serious pancytopenia. Oral ulcerative lesions which probably provided a portal of entry for the organisms were found in both patients. Ulcerations in the oral cavity and upper respiratory tract of patients with leukemia ${ }^{23-28)}$ are known to be caused by the leukemic infiltration, by the toxic effect of cytostatic preparations, particularly antimetabolites, by necrotic processes at sites of hemorrhages and hematomas, by fungal implantation, by viral infection, mainly due to Herpes hominis virus, and also by aphthous processes of unknown etiology, and further aggravated by bacterial intervention. In both cases, F. nucleatum, which is constantly present in large numbers in the mouth ${ }^{17}$, was inferred to have infected the oral ulcerative lesions and gained access to the general circulation, taking advantage of depressed host resistance.

In our patients, the pathogenic role of F. nucleatum was suggested by the fact that the isolation of this organism was made from at least 2 separate blood cultures in accordance with clinical symptoms enough to suspect septicemia.

The clinical features of fusobacterial septicemia observed in these cases seemed to be similar to those of sepsis due to any aerobic or facultative gram-negative rod. Evidence of thrombophlebitis or embolic phenomena, which occurs frequently in the Bacteroides species septicemia ${ }^{4)}$, or metastatic abscesses was lacking, probably because our patients were promptly treated with antimicrobial agents which proved to be active against the isolates.

Many anaerobic bacteria have roughly predictable patterns of antimicrobial susceptibility according to their species $^{7,299}$. With regard to susceptibility of clinical isolates of the Fusobacterium species to commonly employed antimicrobial agents, penicillin $G$ is the most active, lincomycin, clindamycin, chloramphenicol and tetracycline have good activity, erythromycin has poor activity, and the aminoglycosides are unsatisfactory even in very high concentrations $^{29}$. In general, ampicillin and cephaloridine are roughly comparable to penicillin $G$ in activity ${ }^{29}$. In addition, the change in antimicrobial susceptibility of the fusobacterial isolates has not been observed up to the present, while an appreciable number of recent isolates of $\mathrm{B}$. fragilis have been shown to be resistant to the drugs which were once regarded as drugs of choice $^{29)}$.

ACKNOWLEDGEMENT: We wish to express our thanks to Drs $\mathrm{H}$ Kodo and E Kato, under whose care the patients were admitted, for their kind permission to publish this report.

\section{REFERENCES}

1) Bornstein DL, et al: Anaerobic infectionsreview of current experience. Medicine 43 : 207, 1964.

2) Gelb AF, Seligman SJ: Bacteroidaceae bacteremia: effect of age and focus of infection upon clinical course. JAMA 212: 1038, 1970.

3) Sinkovics JG, Smith JP: Septicemia with Bacteroides in patients with malignant disease. Cancer 25: 663, 1970.

4) Felner JM, Dowell VR, Jr: "Bacteroides" bacteremia. Amer J Med 50: 787, 1971.

5) Wilson WR, et al: Anaerobic bacteremia. Mayo Clin Proc 47: 639, 1972.

6) Chow AW, Guze LB: Bacteroidaceae bacteremia: clinical experience with 112 patients. Medicine 53: 93, 1974.

7) Garrod LP: Sensitivity of four species of Bacteroides to antibiotics. Brit Med J 2: 1529, 1955.

8) Tynes BS, Utz JP: Fusobacterium septicemia. Amer J Med 29: 8'79, 1960.

9) Murphy R, et al: Fusobacterium septicemia following a human bite. Arch Intern Med 111 : 51, 1963.

10) Robinow M, Simonelli FA: Fusobacterium bacteremia in the newborn. Amer $J$ Dis Child 110: 92, 1965.

11) Cowan ST, Steel KJ: Manual for the Identification of Medical Bacteria. Cambridge University Press, Cambridge, 1970.

12) Holdeman LV, Moore WEG (Eds): Anaerobe Laboratory Manual. Virginia Poly- 
technic Institute Anaerobe Laboratory, Blacksberg, Va., 1972.

13) Ninomiya K, et al: Simple and expedient methods of differentiation among Bacteroides, Sphaerophorus and Fusobacterium. Jap J Med Sci Biol 25: 63, 1972.

14) Ninomiya $K$ : Fusobacterium nucleatum. Med Technol 4: 441, 1976 (in Japanese).

15) Bartlett JG, et al: The bacteriology of aspiration pneumonia. Amer J Med 56: 202 1974.

16) Bartlett JG, Finegold SM: Anaerobic infections of the lung and pleural space. Amer Rev Resp Dis 110: 56, 1974.

17) Buchanan RE, Gibbons NE (Eds.): Bergey's Manual of Determinative Bacteriology. 8th ed. Williams and Wilkins Co., Baltimore, 1974.

18) Wilson GS, Miles AA: Topley and Wilson's Principles of Bacteriology, Virology and Immunity. 6th ed. E Arnold, London, 1975.

19) Washington JA, II : Comparison of two commercially available media for detection of bacteremia. Appl Microbiol 22: 604, 1971.

20) Ellner PD, et al: Recovery and identification of anaerobes: a system suitable for the routine clinical laboratory. Appl Microbiol 26: 904,
1973.

21) Loesche $\mathrm{WJ}$ : Oxygen sensitivity of various anaerobic bacteria. Appl Microbiol 18: 723, 1969.

22) Klainer AS, Beisel WR: Opportunistic infection: A review. Amer J Med Sci 258: 431, 1969.

23) Armstrong $D$, et al: Infectious complications of neoplastic disease. Med Clin N Amer 55: 729, 1971.

24) Hitchings GH, Rhoads GP : 6-Mercaptopurine. Ann New York Acad Sci 60: 183, 1954.

25) Adour KK: Oral manifestations of systemic disease. Med Clin N Amer 50: 361, 1966.

26) Viola MV: Acute leukemia and infection. JAMA 201: 103, 1967.

27) Wintrobe MM: Clinical hematology. 6th ed. Asian ed. Lea and Febiger, Phila., 1967/ Igaku Shoin, Tokyo, 1967.

28) Skurkovich GV: Lesions in the upper respiratory tract in blood dyscrasias. John Wiley and Sons, New York, 1973.

29) Okada J, et al: Changes of antimicrobial susceptibility of anaerobic bacteria from clinical specimens. Jap J Antibiotics 28 : 727, 1975 (in Japanese). 